\title{
Design of Animal Myocardial Contractile Force Detection System Based on Tissue Engineering
}

\author{
Guiqing $\mathrm{Xi}^{1}$, Ke $\operatorname{Han}^{2}$, Ming Zhao ${ }^{2}$, Caojun Huang ${ }^{1}$, and Feng $\operatorname{Tan}^{1}$ \\ ${ }^{1}$ College of Information and Technology Heilongjiang Bayi Agricultural University Daqing \\ 163319, China \\ ${ }^{2}$ Harbin University of Commerce, Haerbin 150028, China \\ xiguiqing@163.com
}

\begin{abstract}
This paper uses the sensor convert animal myocardial contractile force into a voltage signal, which is collect by a MCU acquisition, transferred to the computer, using the PC visualization language VB compiled a data acquisition and processing platform, collected data can be stored into a computer, and real-time curve of the data changes is plotted at any time. Through testing the tissue engineered cardiac tissue strips micro contractility, it was proved that the platform operation is of stable performance, accurate data acquisition, processing method effective, the system can also be applied in other similar signal detection and acquisition and other fields.
\end{abstract}

Keywords: tensile testing, data acquisition, tissue engineering, real-time plotting.

\section{Introduction}

Tissue engineering is a life science emerging in the $1980 \mathrm{~s}$, and the structure of tissueengineered cardiac muscle tissue is an important aspect of tissue engineering. Now after mix-culture of the collagen and myocardial cells can obtain a consistent beat, the beating frequency coincide that of the natural growth of myocardial tissue. But myocardial beat generation of contractile force is also an important indicator tested in tissue-engineered myocardial performance, however the accurate detection of the contractile force method is still not mature enough.

The computer acquisition of the real-time analog signal has a relatively mature technology. The data collection and transmission mainly use the A/D data acquisition card and the existing communication protocols (such as RS232/485)[1]. So how to utilize the existing high-level language to design data operation and processing platform with many functions is a key issue.

This article starts from the device of contractile force detection, and then designs the cantilever stretch detect structure. Using MCU collects force signals from the sensor and transmits to the computer. Based on the implementation of data acquisition, use VB language to design the myocardial contraction operation platform which is facilitate to the operation of the data acquisition and the processing function[2]. 


\section{Experiments and Methods Myocardial Contractile Force Detecting Device}

Simple mechanical contraction force stretching machinery should has the advantages of simple structure, good specimen tensile coaxial, convenient assembly and disassembly, and preload adjustment method is reliable, good experimental repeatability etc...According to the above requirements, this paper designs such cantilevered contractile tension detecting device as the Fig.1.

As shown in Fig.1, contractile force detection device is composed of several parts including the pedestal / bracket, force sensor, a pre-load nut and support cantilever beam etc... what's more the tension sensor with temperature compensation is the core part of the device, and the strain gauges were embedded in silicone adhesive and is isolated from the air, avoiding the external corrosion, all the other parts are made of stainless steel, thus the whole device can be placed in water, it is necessary for the measurement of myocardial strip contraction force, because tissue engineered cardiac tissue strips stays in culture liquid and if left exposed in the air, myocardial cells will die soon[3]. Near the bottom of the bracket in somewhere can be folded into 90 degrees so that make sure the myocardial strip in horizontal position, which enables the detection of myocardial placed in the culture dish containing a culture liquid container.

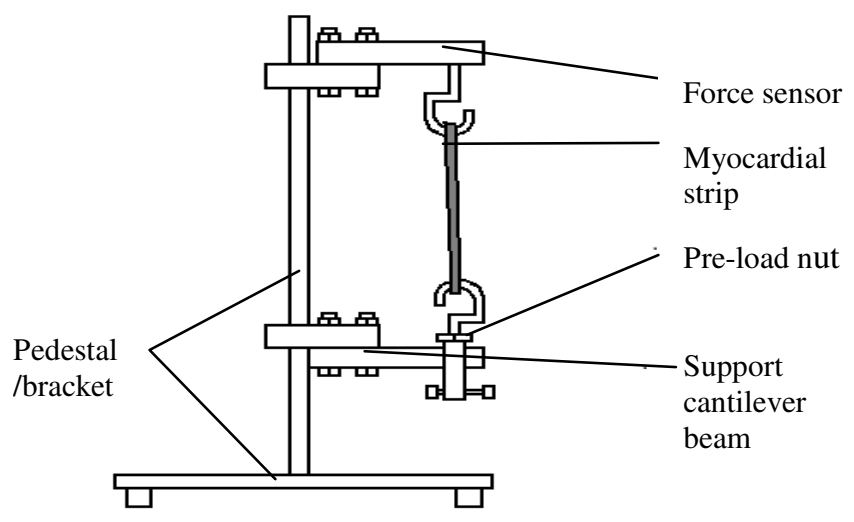

Fig. 1. Schematic of contractile force detecting device

Using the coaxial positioning principle make the sensor and the support cantilever beam are kept in the same plane, which are respectively provided with a hook, the hook through the special structure and maintain strict with the axis, it ensures that the stretching of the strict coaxiality, reduces the test principle error[4]. Through the preloaded nut to be measured myocardial strip preload in the device, then the weak beats of the myocardial strip can be detected by the sensor. 


\section{Computer Data Acquisition System}

Force sensor detects the contractility signal through the sensor converted into a weak electrical signal, through the A/D converter for converting to the digital signal, using SCM acquisition, transmit to the computer ; for storing, printing, modification and analysis processing by the data acquisition and processing platform. Throughout the course of the basic frame diagram shown in Fig.2.

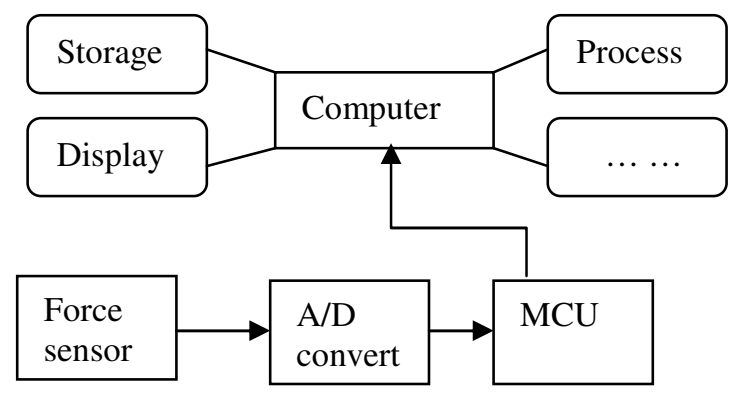

Fig. 2. Frame diagram of data acquisition and processing

\subsection{The Choice of the Sensor}

As a result of myocardial contractile force is very small so it needs to select the micro force measuring sensor, through the experiment choose LDWX-1 type micro tension sensor, it adopts the foil strain gauge affixed to the alloy steel sphere, is subjected to a tensile force, has the advantages of high accuracy, good stability, low temperature drift, good output symmetry, compact structure and measuring micro force characteristics.

\subsection{The Choice of the ADC}

ADC choose AD574, it is launched by the United States of America analog digital company (Analog), 12 bit high speed successive comparative A/D converter, a builtin bipolar circuit hybrid integrated remarkable conversion monolithic, with fewer external components, low power consumption, high precision, and has the advantages of automatic zero adjustment and automatic polarity conversion function, only need small external RC-pieces can constitute a complete A / D converter[5].

\subsection{The Choice of the MCU}

The MCU mainly completes the data collecting, processing, transmitting and receiving orders and other functions; it is the core of the system. As the entire core of the system controller is real-time at work, in order to meet the design requirements, which requires its power consumption must be very low, the speed should be quick and reliable performance. So the controller chooses low power MSP430 microcontroller, with its 
own unique strengths to reducing the chip's supply voltage and a flexible and controllable operating clock and other aspects have its one's own knack in.MSP430 series single chip power supply voltage ranges $1.8-3.6 \mathrm{~V}$. Thus could let the clocking at $1 \mathrm{MHz}$ operating conditions, chip current will be in about $200 \sim 400 \mathrm{uA}$, clock shutdown mode with the lowest power only $0.1 \mathrm{uA}$, other properties include the speed, reliability and other aspects are also consistent with the system requirements.

\section{Host Computer Data Acquisition and Processing Platform Design}

\subsection{The Main Function of the System and the Basic Structure of the Procedure}

Based on the VB language development of testing platform to realize the files reading and writing, data acquisition control and preliminary processing, acquisition of tension values in real-time curve drawing in the plane coordinate system; image recording, image redraw, coordinate axis size transform functions.

This platform program is designed according to several function module structure, simple flow chart as shown in Fig.3, which has a more detail description of the processing function module of the change curve of the image to redraw, the other modules are marked position in the process, their specific design will be introduced step-by-step.

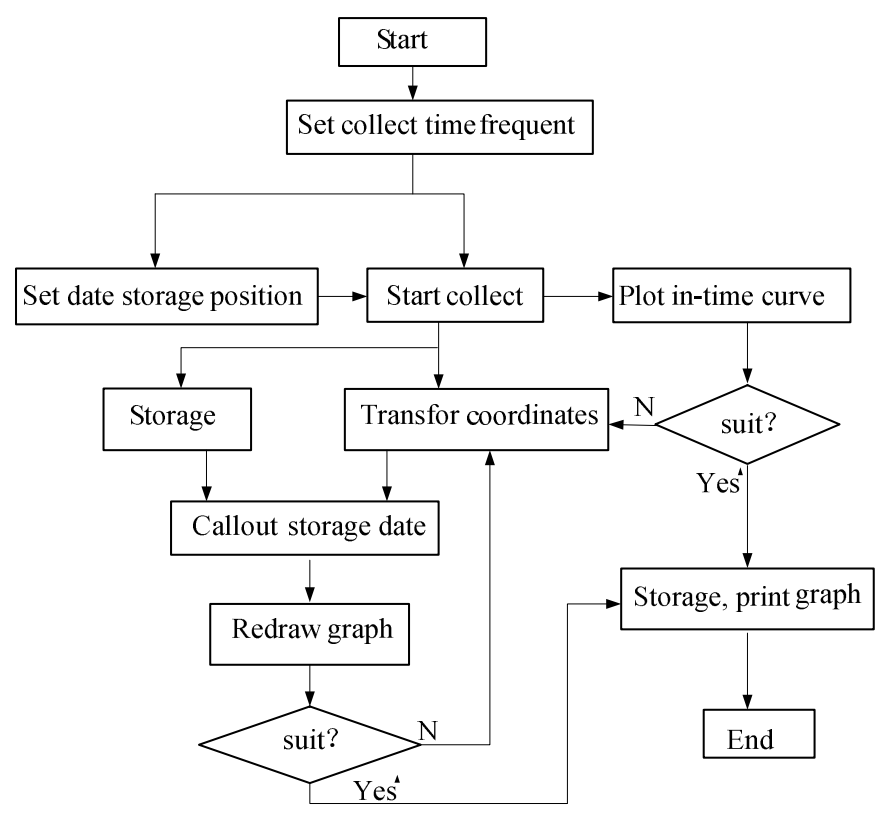

Fig. 3. System program 


\subsection{The Data Acquisition Module Program}

VB program via the serial port control chip utilize the data sent from MCU, storage, and sent out acquisition command etc; before reading the signal, initialize acquisition channel as follows:

Mscomm1.Commport=2

Select COM2

Mscomm1.Settings="9600,N,8,2" // Set the communication parameters

Mscomm1.Inputlen=0 //Read into all of the characters from buffer for receiving

Mscomm1.OutbufferSize $=256 \quad$ // Set the send buffer size

Mscomm1.InbufferSize $=512$ / / Sets the receive buffer size

Mscomm1.PortOpen=True Open COM2

In order to eliminate the error caused by the interference signal in the acquisition process, we designed a continuous acquisition sampling five times for each signal, get the mean value of four significant figures, and so that we can $t$ reduce the interference error to some extent[6].

\subsection{Curve of Real-Time Plotting Module}

Curve of real-time plotting module is the main function of this platform, including real-time display of curves and data collection, tracking and calculation of the value, the time interval between the peak of the window display and mouse click coordinates real-time display and other functions.

The following procedures achieve the real-time plotting data curve function:

Picture1.DrawStyle $=0$ ' //Set resolution for mapping as VB default resolution

Picture1.Line ((m-pinl), yali)-(m, data), curvec //Plot the curve

yali $=$ data

Call draw_axis // Call draw axis function

If the acquisition time is set very long, the curve size will exceed computer screen, then we take the approach that make the screen curve automatically saved, then clear the screen and display the next screen data ,the achieve specific statement as follows:

If $\mathrm{m}>=\mathrm{x} 0$ Then

$\mathrm{k}=\mathrm{k}+1$

SavePicture Picture1.Image, Label5.Caption + CStr(k) + ".bmp" //Full screen to save the picture, called the input file name + label

$\mathrm{m}=0$

Picture1.Cls // Clear the screen redraw

In order to facilitate the data real-time observation, we designed a mouse click on any point of a curve, it will pop up a window marked change point coordinates, allowing users to roughly estimate the numerical size, in order to change the coordinates for the ideal figure drawing. Realize the statement as follows:

$\mathrm{t}=$ Format $(\mathrm{x}$, "fixed // Get the value of time axis

press $=$ Format(y, "fixed")' //Get the value of pressure axis Coordinate display

Show' // Window displays the value [7]. 


\subsection{Coordinate Transformation and Redraw Graphics Module}

The transformation of coordinates for adapting to the different size tension measurement value mapping, in the measurement of tissue engineered cardiac tissue strips that tension measurement value very small sample, we need a coordinate scale which is relatively concentrated, and so we use section method for drawing or redrawing curve.

Sensor measuring range is $0 \sim 5 \mathrm{~N}$, then we will divide coordinate axis into 3 types. one measuring range is more than $2 \mathrm{~N}$, its coordinates minimum scale is 0.5 ; a force measurement in the range of $0.2 \sim 2 \mathrm{~N}$, its scale is 0.1 ; the other is a force measurement range less than $0.2 \mathrm{~N}$, the corresponding coordinate minimum scale is 0.01 .Start is the system default first coordinate mode[8]. In order to allow users to pay attention to selection of coordinate system, the system doesn't draw the coordinates, but in need of drawing graphics that it prompts the user to choose the coordinate system.

The main program calls the appropriate code to change the current drawing coordinate system, so as to redraw graphics, it can also change graphics that have been drawn .The following figure is a representation of the function of the specific effect. From the Fig. $4 \sim 6$ comparison, we can see in Fig.5, expressing data peak interval and familiar change trend more clearly, so using this method solves the appropriate display of graphics[9].

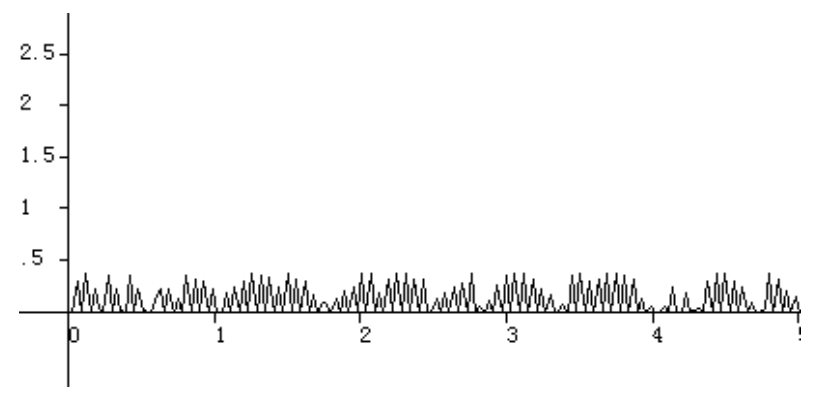

Fig. 4. Coordinate drawn in the range more than $2 \mathrm{~N}$ data curves

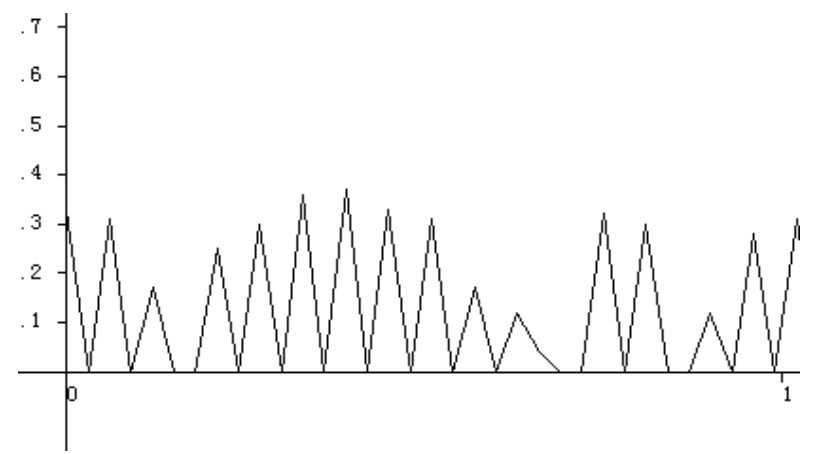

Fig. 5. Coordinate drawn in the range $0.2 \sim 2 \mathrm{~N}$ data curves (abscissa elongated 5 times) 


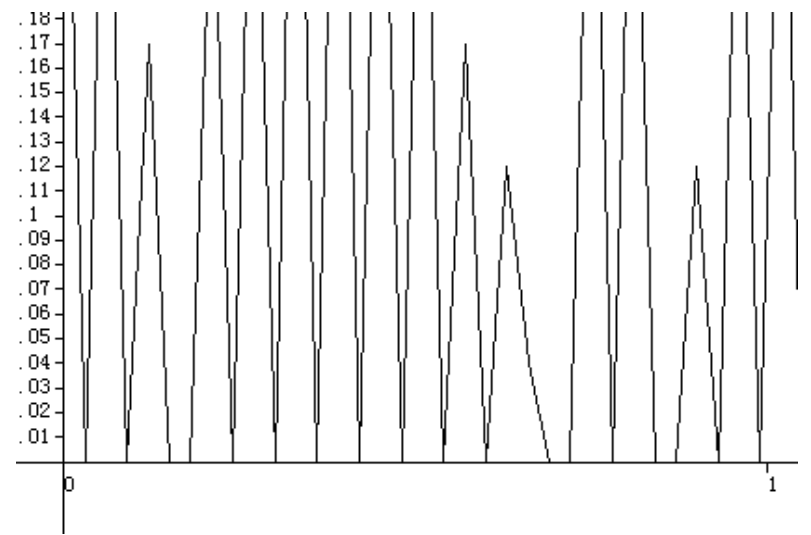

Fig. 6. Coordinate drawn in the range less than $0.2 \mathrm{~N}$ data curves (abscissa elongated 5 times)

Of course, the graphics processing not only these features, but also including the display of the curve of the past, amplification, modify and so on, so the operation of the graphics module actually also has some of the features that of image viewing software.

\section{$5 \quad$ Meaus of Function Modules}

The data acquisition platform written by VB language, which comprises a very eyecatching data curve drawing area, while other regions is a drop-down menu and the corresponding icon in the menu, the menu can achieve some process control and data processing function.

(1)File menu: File menu containing the control of the measurement process, including measurement start, pause break, end and exit of the measure and some useful control functions; also including the initialization of measurement, setting sampling time and frequency of sampling.

(2)Processing menu: The menus includes calculation and display of data values of the maximum and minimum, as well as redraw graphics coordinate value input interface and so on.

(3)Curve menu: curve menu is mainly some processing for the curves, including the display of real-time curve, saving the current curve, enlarging the current curve, printing the current curve, showing the past curve and the curve itself and set the background color.

(4)Help menu: Help menu links an introduction text about system using method and matters needing attention to, which can provides the user with the necessary tips and help. 


\section{Conclusion}

This paper introduces the application of VB and microcontroller used in the development of tissue engineering cardiac tissue contraction force measuring system. After the test results showing that the system has good stability, wide measuring range, data processing and high drawing function. This system has good portability, and can be applied to many similar systems. It provides a convenient operation, accurate data system in some experimental data testing for tissue engineering.

Acknowledgment. Funds for this research was provided by Science and technology research Projects by Educational Commission of Heilongjiang Province of China (12531154).

\section{References}

1. Fang., W., et al.: Cardiac excitation contraction coupling in mammalian development in postnatal changes. Progress in Physiological Sciences 44(3), 227-232 (2013)

2. Lüs, L.S., He, W., et al.: Bioreactor cultivation enhances NTEB formation and differentiation of NTES cells into cardiomyocytes. Cloning and Stem Cells (2008)

3. Jian-sheng, W., Jian-meng, W.: Biomedical Signal Detection and Processing. Time Education (9), 6-7 (2011)

4. Lo, L., et al.: Design of Weak Signal Detection Based on MSP430G2452. Industrial Control Computer 26(5), 15-16 (2013)

5. Jianhua, W., et al.: Development of steel strand tension measurement sensor. Transducer and Microsystem Technologies 29(5), 83-86 (2010)

6. Guiyun, X., et al.: Development of high speed data acquisition system based on Vibration Analysis. Experimental Technology and Management 30(7), 47-50 (2013)

7. Kai, X., Jian, Z.: MSP430 series single-chip microcomputer system project design and practice, pp. 3-6. Mechanical Industry Press, Beijing (2009)

8. Yongqiang, S.: The realization way of the data acquisition in the VB environment. China Auto Industry (2004)

9. Yang, L.: Design of pressure sensor data acquisition system PC software based on VB. Mechanical Engineer (12), 56-58 (2012)

10. ZHanming, L., et al.: Design of data acquisition system based on VB and Advantech data acquisition card. Computer and Modernization (7), 236-238 (2012) 